\title{
1 Substrate and electron donor limitation induce activities in a green sulphur bacterium
}

4

5 Running title: Limitation induces phenotypic heterogeneity

6

7 Zimmermann, M. ${ }^{1,2}$, Escrig, S. ${ }^{3}$, Lavik, G. ${ }^{5}$, Kuypers, M.M.M. ${ }^{5}$, Meibom, A. ${ }^{3,4}$,

8 Ackermann, M. ${ }^{1,2}$, and Schreiber, F. ${ }^{1,2,6}$

9

10 'Department of Environmental Systems Science, ETH Zurich - Swiss Federal Institute of Technology,

11 Zurich, Switzerland.

12 'Department of Environmental Microbiology, Eawag - Swiss Federal Institute of Aquatic Science and 13 Technology, Dübendorf, Switzerland.

14 '3aboratory for Biological Geochemistry, School of Architecture, Civil and Environmental Engineering 15 (ENAC), École Polytechnique Fédérale de Lausanne (EPFL), Lausanne, Switzerland.

$16{ }^{4}$ Center for Advanced Surface Analysis, Institute of Earth Sciences, University of Lausanne, Lausanne, 17 Switzerland.

$18{ }^{5}$ Department of Biogeochemistry, Max Planck Institute for Marine Microbiology, Bremen, Germany

19 'Division Biodeterioration and Reference Organisms, Department of Materials and Environment, BAM

20 - Federal Institute for Materials Research and Testing, Berlin, Germany.

Correspondence: Matthias Zimmermann, Eawag, Seestrasse 79, 6047 Kastanienbaum, Switzerland, email: matthias.zimmermann@eawag.ch

24

25 Frank Schreiber, BAM, Unter den Eichen 87, I2205 Berlin, Germany email: frank.schreiber@bam.de

This document is the accepted manuscript version of the following article:

Zimmermann, M., Escrig, S., Lavik, G., Kuypers, M. M. M., Meibom, A., Ackermann, M., \& Schreiber, F. (2018). Substrate and electron donor 1imitation induce phenotypic heterogeneity in different metabolic activities in a green sulphur bacterium. Environmental Microbiology Reports, $10(2), 179-183$.

https://doi .org/10.1111/1758-2229.12616 
Abstract

Populations of genetically identical cells can display marked variation in phenotypic traits; such variation is termed phenotypic heterogeneity. Here we investigate the effect of substrate and electron donor limitation on phenotypic heterogeneity in $\mathrm{N}_{2}$ and $\mathrm{CO}_{2}$ fixation in the green sulphur bacterium Chlorobium phaeobacteroides. We grew populations in chemostats and batch cultures and used stable isotope labelling combined with nanometer-scale secondary ion mass spectrometry (NanoSIMS) to quantify phenotypic heterogeneity. Experiments in $\mathrm{H}_{2} \mathrm{~S}$ (i.e. electron donor) limited chemostats show that varying levels of $\mathrm{NH}_{4}{ }^{+}$limitation induce heterogeneity in $\mathrm{N}_{2}$ fixation. Comparison of phenotypic heterogeneity between chemostats and batch (unlimited for $\mathrm{H}_{2} \mathrm{~S}$ ) populations indicates that electron donor limitation drives heterogeneity in $\mathrm{N}_{2}$ and $\mathrm{CO}_{2}$ fixation. Our results demonstrate that phenotypic heterogeneity in a certain metabolic activity can be driven by different modes of limitation and that heterogeneity can emerge in different metabolic processes upon the same mode of limitation. In conclusion, our data suggest that limitation is a general driver of phenotypic heterogeneity in microbial populations.

Keywords: Phenotypic variation, phenotypic heterogeneity, phenotypic diversity, NanoSIMS, nutrient limitation, Lago di Cadagno, Chlorobium phaeobacteroides, dinitrogen fixation, carbon dioxide fixation 
52 Phenotypic heterogeneity is a widespread phenomenon manifesting itself in

53 fundamental microbial traits such as antimicrobial persistence (Balaban et al., 2004),

54 competence for DNA uptake (Maamar et al., 2007), chemotaxis (Emonet and Cluzel,

55 2008), and metabolic activity (Ozbudak et al., 2004; Kiviet et al., 20I4; Kotte et al.,

56 20I4; New et al., 20I4; Solopova et al., 20I4; Schreiber et al., 2016). It has been

57 shown that phenotypic heterogeneity is an evolvable microbial trait because it is

58 genetically controlled (Ozbudak et al., 2002). Phenotypic heterogeneity helps

59 microbial populations (Ackermann, 2015) to adapt to fluctuating environmental

60 conditions (Balaban et al., 2004; Kussell and Leibler, 2005; Acar et al., 2008;

61 Beaumont et al., 2009; Ratcliff and Denison, 2010; Arnoldini et al., 20I4; Schreiber et

62 al., 2016), aids in the division of labour within isogenic cell populations (Ackermann

63 et al., 2008), and can result from negative frequency-dependent interactions in mixed

64 resource environments (Healey et al., 2016). Multiple studies on phenotypic

65 heterogeneity have been conducted with microbial model strains, while only a few

66 studies have investigated environmental isolates (Ziv et al., 20I3; Holland et al., 20I4;

67 New et al., 2014; Miot et al., 2015; Guantes et al., 2016) or natural microbial 68 populations (Zimmermann et al., 2015; Sheik et al., 20I5; Kopf et al., 20I5b). Thus,

69 there remains a knowledge gap as to how phenotypic heterogeneity is controlled in 70 environmental bacteria without long laboratory culture history and in natural 71 microbial populations.

73 While the molecular mechanisms that generate phenotypic heterogeneity have 74 received considerable attention, it remains unclear how the nutrient environment of 
75 a population affects phenotypic heterogeneity. A recent study showed that

76 heterogeneity in $\mathrm{N}_{2}$ fixation is induced by the level of $\mathrm{NH}_{4}^{+}$limitation in the 77 heterotrophic model organism Klebsiella oxytoca (Schreiber et al., 2016). This study 78 investigated $\mathrm{N}_{2}$ fixation heterogeneity in glucose-limited and $\mathrm{N}_{2}$-saturated 79 chemostats with varying degrees of $\mathrm{NH}_{4}^{+}$supply (from depletion to limitation to 80 saturation). It was shown that the closer $\mathrm{NH}_{4}{ }^{+}$limitation approached the transition 81 point between limitation and saturation the higher the heterogeneity in $\mathrm{N}_{2}$ fixation 82 (Schreiber et al., 2016). However, it remained untested if other types of limitation 83 except those of $\mathrm{NH}_{4}^{+}$can induce heterogeneity in $\mathrm{N}_{2}$ fixation or if heterogeneity 84 occurs in metabolic activities other than $\mathrm{N}_{2}$ fixation. Furthermore, it was not tested 85 in the previous study if $\mathrm{NH}_{4}^{+}$limitation also affects heterogeneity in physiologically 86 and phylogenetically distant $\mathrm{N}_{2}$ fixing bacteria. Here, we investigated phenotypic heterogeneity in $\mathrm{N}_{2}$ and $\mathrm{CO}_{2}$ fixation with stable isotope labelling combined with NanoSIMS imaging in the green sulphur bacterium $C$. phaeobacteroides. NanoSIMS measures the isotopic ratios at single cell resolution 91 (Musat et al., 2012). It allows to quantify the incorporation rate of anabolic 92 substrates on the single-cell level when combined with feeding isotopically labelled 93 substrates for a part of the generation time of the cells. The strain was freshly 94 isolated from the chemocline of the meromictic lake Lago di Cadagno situated in 95 Ticino, Switzerland (Zimmermann et al., 20I5) and went through minimal cycles of 96 growth in the laboratory before experimentation. C. phaeobacteroides performs 97 anoxygenic photosynthesis under strictly anaerobic conditions with $\mathrm{H}_{2} \mathrm{~S}$ as electron 98 donor, grows single-celled, but can also form short (approx. 2-5 cells) filaments. It 99 fixes $\mathrm{CO}_{2}$ as a carbon source and $\mathrm{N}_{2}$ (if limited and depleted for $\mathrm{NH}_{4}^{+}$) as a nitrogen 
source. We investigated $C$. phaeobacteroides because previous work indicated that it

101 displays phenotypic heterogeneity in $\mathrm{N}_{2}$ fixation in its natural habitat (Halm et al.,

102 2009; Zimmermann et al., 2015). In addition, previous work showed that $\mathrm{N}_{2}$ fixing,

103 phototrophic cyanobacteria living in a microbial mat also display pronounced levels

104 of phenotypic heterogeneity (Woebken et al., 20l4). It should be noted that in

105 those two studies phenotypic heterogeneity can also be the result of genetic

106 differences in these natural populations or could be induced by environmental

107 heterogeneity.

\section{Results and Discussion}

110 We grew C. phaeobacteroides populations in chemostat and batch culture to

111 disentangle two types of limitation. Chemostats were $\mathrm{H}_{2} \mathrm{~S}$ (i.e. electron donor)

112 limited and were experimentally varied in the level of $\mathrm{NH}_{4}^{+}$(i.e. substrate) limitation.

113 This was achieved by changing the supply of $\mathrm{NH}_{4}^{+}$in the feed medium from saturated

114 to fully depleted while supplying saturating amounts of $N_{2}$ gas (Figure la). In the

115 range of $\mathrm{NH}_{4}^{+}$limitation, cells exhaust all the supplied $\mathrm{NH}_{4}^{+}$because $\mathrm{NH}_{4}^{+}$

116 assimilation is preferred over energetically more expensive $N_{2}$ fixation. Hence, the

117 amount of $\mathrm{N}_{2}$ that cells fix depends on the $\mathrm{NH}_{4}^{+}$supply (Supplementary Table I). In

118 contrast, exponential batch cultures were completely unlimited for both electron

119 donor and substrates. Chemostat and batch populations were pulse-fed with ${ }^{15} \mathrm{~N}_{2}$

120 and ${ }^{13} \mathrm{CO}_{2}$ and analysed for single-cell incorporation with NanoSIMS (Figure Ib).

121 Hence, by comparing cell-to-cell heterogeneity using the coefficient of variation (CV)

122 of chemostat-grown populations with batch-grown populations in the absence of

$123 \mathrm{NH}_{4}^{+}$the effect of electron donor (i.e. $\mathrm{H}_{2} \mathrm{~S}$ ) limitation on two different metabolic

124 activities $\left(\mathrm{N}_{2}\right.$ fixation and $\mathrm{CO}_{2}$ fixation) can be deduced. 
a

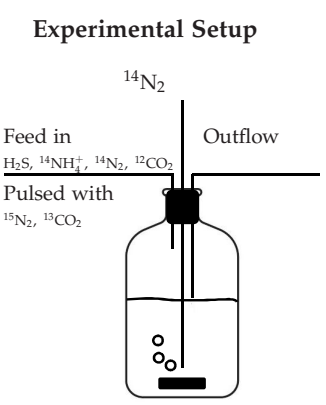

b

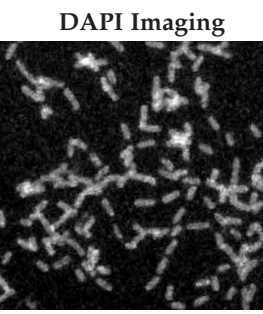

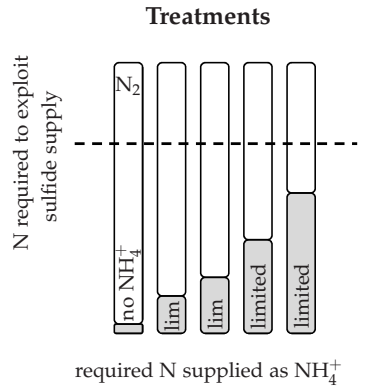

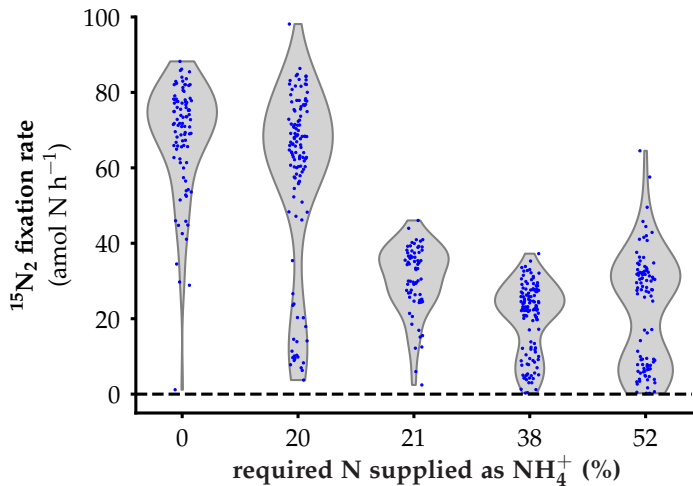

d
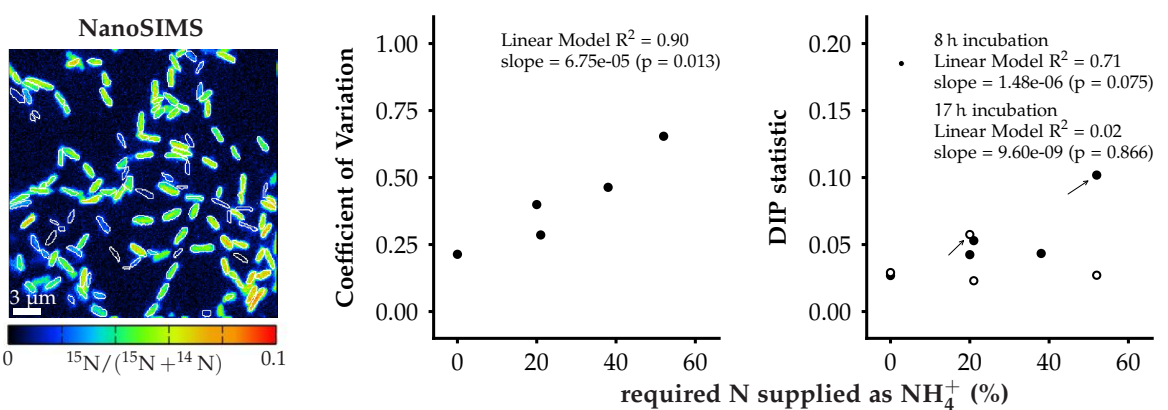

Figure I. $\mathrm{NH}_{4}^{+}$limitation induces $\mathrm{N}_{2}$ fixation heterogeneity at the single-cell level in chemostatgrown $C$. phaeobacteroides populations. (a) Varying levels of $\mathrm{NH}_{4}^{+}$limitation in the presence of excess $\mathrm{N}_{2}$ was realized in chemostats. Chemostats were operated under $\mathrm{N}_{2}$ atmosphere with a constant supply of feed medium with varying $\mathrm{H}_{2} \mathrm{~S}: \mathrm{NH}_{4}{ }^{+}$-ratios (volume $=30 \mathrm{ml}$; dilution rate $=0.02 \mathrm{~h}^{-1}$ ). After 10 days of equilibration (five volume exchanges), the chemostats were incubated with a pulse of ${ }^{15} \mathrm{~N}_{2}$ and ${ }^{13} \mathrm{CO}_{2}$ for 8 hours (23\% of the generation time) or 17 hours (50\% of the generation time). (b) Example of DAPI total fluorescence image and corresponding NanoSIMS measurement for ${ }^{15} \mathrm{~N} /\left({ }^{14} \mathrm{~N}+{ }^{15} \mathrm{~N}\right)$ ratio in single $C$. phaeobacteroides cells. (c) ${ }^{15} \mathrm{~N}_{2}$ fixation rates of single cells (blue dots) for different levels of $\mathrm{NH}_{4}^{+}$limitation. The kernel probability density is plotted in grey with a constant maximum width. (d) Coefficients of variation increase with decreasing $\mathrm{NH}_{4}^{+}$limitation $(\mathrm{p}=0.0 \mathrm{I})$. Bimodality in $\mathrm{N}_{2}$ fixation at varying levels of $\mathrm{NH}_{4}^{+}$limitation evaluated by the Hartigan's DIP statistic. Increasing values indicate increasing deviation from unimodality. The significance and the magnitude of the correlation between decreasing $\mathrm{NH}_{4}{ }^{+}$supply and increasing bimodality is stronger after 8 hours of incubation (black circles, $p=0.075$ ) as compared to 17 hours of incubation (open circles, $p=0.866$ ). Approximated $p$-values $<0.05$ for the Hartigan's DIP statistic are indicated with an arrow. See Supplementary table I for the number of measured cells and tabulated values for each experimental condition. Note that two of the levels of the required $\mathrm{N}$ supplied as $\mathrm{NH}_{4}^{+}(20 \%$ and $21 \%)$ are close to each other, and that the difference between these two experiments might not be consistently replicated in future experiments.

The experiments showed that $\mathrm{NH}_{4}^{+}$limitation induces heterogeneity, expressed as the coefficient of variation, in $\mathrm{N}_{2}$ fixation (Figure Ic and d). Heterogeneity increased the closer $\mathrm{NH}_{4}^{+}$limitation approached the transition point between limitation and saturation. These results are consistent with a previous study on the heterotrophic $\mathrm{N}_{2}$ fixer K. oxytoca (Schreiber et al., 2016) and show that phenotypic heterogeneity of 
152 the same activity (i.e. $\mathrm{N}_{2}$ fixation), driven by the same mode of limitation $\left(\mathrm{NH}_{4}^{+}\right.$

153 limitation), is induced in the same way in physiologically and phylogenetically distant

154 bacterial species with different laboratory culture histories.

155

156 Increasing $\mathrm{NH}_{4}{ }^{+}$supply led to a bimodal distribution in $\mathrm{N}_{2}$ fixation activities in the 157 population as shown by an increasing Hartigan Dip Statistic (i.e. increasing deviation 158 from unimodality) after $8 \mathrm{~h}$ of isotopic labelling (Figure Ic and d). The relationship 159 between bimodality and $\mathrm{NH}_{4}^{+}$supply weakened upon $17 \mathrm{~h}$ incubation times with 160 stable isotopes (Figure Id) indicating that cells with high initial rates tended to lower 161 their $\mathrm{N}_{2}$ fixation rate and cells with low initial activity increased their rate within the 162 duration of the incubation. The generation time $(34.7 \mathrm{~h})$ set by the dilution rate was 163 lower than the stable isotope incubation time indicating that cells switch between 164 high and low $\mathrm{N}_{2}$ fixation rate within their cell cycle.

166 Next, we asked if limiting cells experimentally by a different substrate also affects 167 phenotypic heterogeneity. We chose to limit cells with $\mathrm{H}_{2} \mathrm{~S}$, the central electron 168 donor for phototrophic growth of $C$. phaeobacteroides in our medium. Populations 169 grown in chemostats are $\mathrm{H}_{2} \mathrm{~S}$-limited, while populations grown in batch are 170 unrestricted of any substrate including $\mathrm{H}_{2} \mathrm{~S}$. Comparison of phenotypic heterogeneity 171 between $\mathrm{NH}_{4}{ }^{+}$-depleted batch- and chemostat-grown populations revealed that $\mathrm{H}_{2} \mathrm{~S}$ 172 limitation induces heterogeneity in $\mathrm{N}_{2}$ and in $\mathrm{CO}_{2}$ fixation (Figure 2). The CV's for $173 \mathrm{~N}_{2}$ and $\mathrm{CO}_{2}$ fixation are significantly different between $\mathrm{NH}_{4}^{+}$-depleted chemostat 174 populations and $\mathrm{NH}_{4}{ }^{+}$-depleted batch populations (Figure $2 \mathrm{~b}$, d; two sample t-test, $\left.175 \mathrm{P}\left(\mathrm{N}_{2}\right)=0.0018, \mathrm{P}\left(\mathrm{CO}_{2}\right)=0.0034\right)$. The two sample t-test compares a single 176 measurement from the chemostat experiment with four replicates form batch 


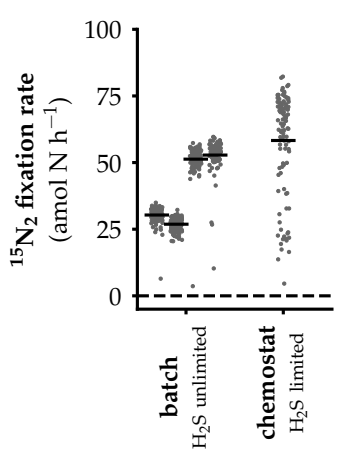

b

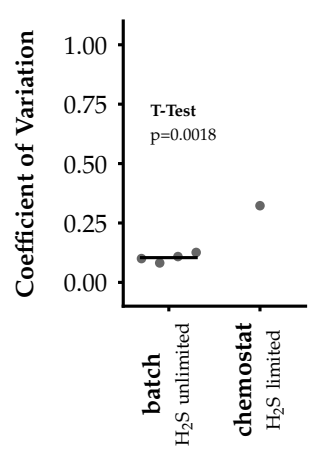

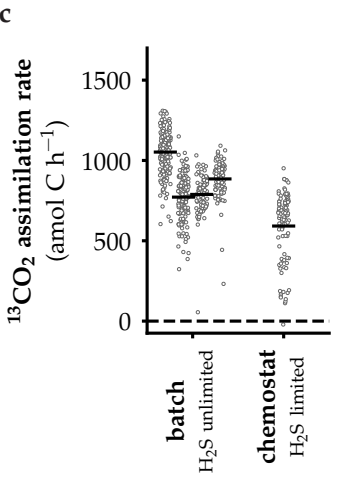

d

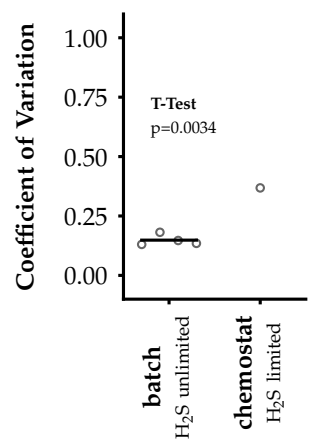

Figure 2. $\mathrm{H}_{2} \mathrm{~S}$ limitation during chemostat incubations induces heterogeneity in single-cell $\mathrm{N}_{2}$ and $\mathrm{CO}_{2}$ fixation activity as compared to unlimited batch-grown C. phaeobacteroides populations. Batch and chemostat incubations were both conducted under $\mathrm{NH}_{4}{ }^{+}$depleted conditions. (a) ${ }^{15} \mathrm{~N}_{2}$ fixation rates of single cells (grey dots) for batch (4 replicates) and chemostat (I replicate) incubations. Average rates are indicated by a black bar. (b) Coefficients of variation (CV) of ${ }^{15} \mathrm{~N}_{2}$ fixation rates. The average $\mathrm{CV}$ of the four batch incubations is indicated by a black bar. The CV of the chemostat incubation is significantly different from the CV's of the four batch incubations (two sample t-test). (c) Calculated ${ }^{13} \mathrm{CO}_{2}$ fixation rates of single cells (grey circles) for batch (4 replicates) and chemostat (I replicate) incubations. Average rates are indicated by a black bar. (d) Coefficients of variation of ${ }^{13} \mathrm{CO}_{2}$ fixation rates. The average $\mathrm{CV}$ of the four batch incubations is indicated by a black bar. The $\mathrm{CV}$ of the chemostat incubation is significantly different form the CV's of the four batch incubations (two sample t-test).

The results indicate that electron donor limitation can induce phenotypic heterogeneity in different metabolic processes within the same bacterial population. Similarly, pronounced heterogeneity has been observed for ${ }^{2} \mathrm{H}_{2} \mathrm{O}$ (growth rate) and ${ }^{15} \mathrm{NH}_{4}^{+}$assimilation in chemostat-grown, carbon-limited Staphylococcus aureus populations (Kopf et al., 20I5a). In combination with the $\mathrm{NH}_{4}{ }^{+}$limitation experiment, the results demonstrate that phenotypic heterogeneity in a certain metabolic activity (i.e. $\mathrm{N}_{2}$ fixation) can be driven by different modes of limitation (here limitation in $\mathrm{NH}_{4}{ }^{+}$and $\mathrm{H}_{2} \mathrm{~S}$ ) in a single microbial population (i.e. C. phaeobacteroides). These

203 results might be best understood in terms of a general feedback between growth 204 state and gene expression (Klumpp et al., 2009; Scott et al., 20 I0; New et al., 20I4; 205 Solopova et al., 20 4; Kotte et al., 20 I4; Guantes et al., 20I6). 
207 Taken together, our results and those of previous studies (Kopf et al., 2015a; 208 Schreiber et al., 2016) suggest that limitation might be a general driver of phenotypic 209 heterogeneity in microbial populations regardless of their culture history, the general 210 physiology of the bacterium, the type of limitation, and the considered metabolic 211 activity. 


\section{Acknowledgements}

213 We thank Thomas Röösli and Hannah Bruderer for their contribution to image

214 analysis, and Gabriele Klockgether (MPI Bremen) for conducting IRMS

215 measurements. The NanoSIMS instrument in the Laboratory for Biological

216 Geochemistry was funded in part by the European Research Council Advanced

217 Grant 246749 (BIOCARB) to A.M. This research was supported by a Leopoldina

218 postdoctoral fellowship (LPDS 2009-42) to FS, a Marie-Curie-Intra-European

219 fellowship for career development (FP7-MC-IEF; 27I929; Phenofix) to FS, and a

220 Synthesis Grant of the ETH Zurich Center for Adaptation to a Changing

221 Environment (ACE) to FS, the Max Planck Society, ETH Zurich and Eawag.

222

\section{Conflict of Interest}

224 The authors do not declare any conflict of interest. 
Acar M, Mettetal JT, van Oudenaarden A. (2008). Stochastic switching as a survival strategy in fluctuating environments. Nat Genet 40: 47I-475. Ackermann M. (20I5). A functional perspective on phenotypic heterogeneity in microorganisms. Nat Rev Microbiol I3: 497-508. Ackermann M, Stecher B, Freed NE, Songhet P, Hardt W-D, Doebeli M. (2008). Selfdestructive cooperation mediated by phenotypic noise. Nature 454: 987-990. Bistable Expression of Virulence Genes in Salmonella Leads to the Formation of an Antibiotic-Tolerant Subpopulation. PLoS Biol I 2: el 001928.

Balaban NQ, Merrin J, Chait R, Kowalik L, Leibler S. (2004). Bacterial Persistence as a Phenotypic Switch. Science (80-) 305: 1622-1625.

Beaumont HJE, Gallie J, Kost C, Ferguson GC, Rainey PB. (2009). Experimental evolution of bet hedging. Nature 462: 90-93.

Emonet T, Cluzel P. (2008). Relationship between cellular response and behavioral variability in bacterial chemotaxis. Proc Natl Acad Sci U S A I 05: 3304-3309.

Guantes R, Benedetti I, Silva-Rocha R, de Lorenzo V. (2016). Transcription factor levels enable metabolic diversification of single cells of environmental bacteria. ISME J 10: II22-II33.

Halm H, Musat N, Lam P, Langlois R, Musat F, Peduzzi S, et al. (2009). Cooccurrence of denitrification and nitrogen fixation in a meromictic lake, Lake Cadagno (Switzerland). Environ Microbiol I I: 1945-1958.

Healey D, Axelrod K, Gore J. (20I6). Negative frequency-dependent interactions can underlie phenotypic heterogeneity in a clonal microbial population.pdf. Mol Syst Biol 12: 877.

Holland SL, Reader T, Dyer PS, Avery S V. (20I4). Phenotypic heterogeneity is a selected trait in natural yeast populations subject to environmental stress. Environ Microbiol 16: 1729-1740.

Kiviet DJ, Nghe P, Walker N, Boulineau S, Sunderlikova V, Tans SJ. (20I4). Stochasticity of metabolism and growth at the single-cell level. Nature 5 I 4: 376-379. Klumpp S, Zhang Z, Hwa T. (2009). Growth Rate-Dependent Global Effects on Gene Expression in Bacteria. Cell I39: I366-1375.

Kopf SH, McGlynn SE, Green-Saxena A, Guan Y, Newman DK, Orphan VJ. (20I5a). Heavy water and I5N labeling with NanoSIMS analysis reveals growth-rate dependent metabolic heterogeneity in chemostats. Environ Microbiol I 7: 2542-2556. Kopf SH, Sessions AL, Cowley ES, Reyes C, Sambeek L Van, Hu Y. (20I5b). Trace incorporation of heavy water reveals slow and heterogeneous pathogen growth rates in cystic fibrosis sputum. e-pub ahead of print, doi: 10.1073/pnas. 15 I2057 I 2. Kotte O, Volkmer B, Radzikowski JL, Heinemann M. (20I4). Phenotypic bistability in Escherichia coli's central carbon metabolism. Mol Syst Biol I 0: I-II.

Kussell E, Leibler S. (2005). Phenotypic Diversity, Population Growth , and Information in Fluctuating Environments. Science (80- ) 309: 2075-2078.

Maamar H, Raj A, Dubnau D. (2007). Noise in gene expression determines cell fate in Bacillus subtilis. Science (80-) 317: 526-529.

Miot J, Remusat L, Duprat E, Gonzalez A, Pont S, Poinsot M. (20I5). Fe biomineralization mirrors individual metabolic activity in a nitrate-dependent $\mathrm{Fe}(\mathrm{II})$ oxidizer. Front Microbiol 6: I-I3.

Musat N, Foster R, Vagner T, Adam B, Kuypers MMM. (20I2). Detecting metabolic activities in single cells, with emphasis on nanoSIMS. FEMS Microbiol Rev 36: 486-5I I. 
275 New AM, Cerulus B, Govers SK, Perez-Samper G, Zhu B, Boogmans S, et al. (20I4).

276 Different levels of catabolite repression optimize growth in stable and variable

277 environments. PLoS Biol I 2: el 001764.

278 Ozbudak EM, Thattai M, Kurtser I, Grossman AD, Oudenaarden A Van. (2002).

279 Regulation of noise in the expression of a single gene. Nat Genet 3 I: 69-73.

280 Ozbudak EM, Thattai M, Lim HN, Shraiman BI, Van Oudenaarden A. (2004).

281 Multistability in the lactose utilization network of Escherichia coli. Nature 427: 737-

28240.

283 Ratcliff WC, Denison RF. (2010). Individual-level bet hedging in the bacterium

284 Sinorhizobium meliloti. Curr Biol 20: 1740-1744.

285 Schreiber F, Littmann S, Lavik G, Escrig S, Meibom A, Kuypers MMM, et al. (2016).

286 Phenotypic heterogeneity driven by nutrient limitation promotes growth in

287 fluctuating environments. Nat Microbiol I: 16055.

288 Scott M, Mateescu EM, Zhang Z, Hwa T. (20I0). Interdependence of Cell Growth

289 Origins and Consequences. Science (80-) 330: 1099-II 02.

290 Sheik A, Muller E, Audinot J-N, Lebrun L, Grysan P, Wilmes P. (20I5). In situ

291 phenotypic heterogeneity among single cells of the filamentous bacterium Candidatus

292 Microthrix parvicella. ISME J I 0: 1274-1279.

293 Solopova A, van Gestel J, Weissing FJ, Bachmann H, Teusink B, Kok J, et al. (20|4).

294 Bet-hedging during bacterial diauxic shift. Proc Natl Acad Sci U S A I I I: 7427-7432.

295 Woebken D, Burow LC, Behnam F, Mayali X, Schintlmeister A, Fleming ED, et al.

296 (20I4). Revisiting N2 fixation in Guerrero Negro intertidal microbial mats with a

297 functional single-cell approach. ISMEJ doi: I0.1038/ismej.20 I4.I44.

298 Zimmermann M, Escrig S, Hübschmann T, Kirf MK, Brand A, Inglis RF, et al. (20I5).

299 Phenotypic heterogeneity in metabolic traits among single cells of a rare bacterial

300 species in its natural environment quantified with a combination of flow cell sorting

301 and NanoSIMS. Front Microbiol 6: I-II.

302 Ziv N, Siegal ML, Gresham D. (20I3). Genetic and nongenetic determinants of cell

303 growth variation assessed by high-throughput microscopy. Mol Biol Evol 30: 2568-

3042578.

305

306 